\author{
G.L. Katkeeva, G. Burkitseterkyzy, Yu.P. Morozov, E.M. Zhunussov \\ Zh. Abishev Chemical-Metallurgical Institute, Karaganda, Kazakhstan \\ (E-mail: gulmarzhan.94@mail.ru)
}

\title{
Thermodynamic analysis of oxidized copper minerals interaction with modified reagent
}

\begin{abstract}
Practicality of preliminary sulphidization of oxidized copper minerals for the purpose of oxidized ore concentration is shown in the paper. For the first time the thermodynamic analysis was carried out for interaction of modified sulphidizing reagent with major components of oxidized copper ore - malachite, azurite and chrysocolla. Ammonium polysulfide formed in the solution of sodium polysulfide and ammonium sulfate was used as modified sulphidizing reagent. Temperature dependence of standard Gibbs energy for the reaction of ammonium polysulfide with oxidized copper minerals was determined. It was noted that standard Gibbs energy for malachite and azurite interaction with modified reagent has negative values in the temperature range of 298,15$373 \mathrm{~K}$, thus showing high probability of interaction, which grows together with temperature. Interaction with chrysocolla is less active than with malachite and azurite but is still possible at mentioned temperature, with interaction probability again proportional to temperature. Thus, the possibility of oxidized copper minerals sulphidization with modified sulphidizing reagent is determined in the research.
\end{abstract}

Keywords: oxidized minerals, chrysocolla, malachite, azurite, modified sulphidizing reagent, sulphidization, thermodynamic analysis, Gibbs energy.

\section{Introduction}

Cheap flotation enrichment methods, conventional for sulfide ore, are less effective for oxidized ore due to natural hydrophilicity of oxidized copper minerals surface [1-3].

One of feasible solutions is chemical modification of minerals via sulphidization resulting in transformation of oxidized minerals into sulfidized ones, which can improve the flotation enrichment efficiency [4-9].

The thermodynamic analysis of possible reactions with determination temperature dependence of standard Gibbs energy has been carried out in order to assess the possibility of sulphidization with modified reagent.

Ammonium polysulfide formed in the solution of sodium polysulfide and ammonium sulfate was used as modified sulphidizing reagent.

The objective of work was to find the temperature dependence of ammonium polysulfide interaction with major components of oxidized copper ore, such as malachite, azurite and chrysocolla.

\section{Experimental}

Three types of interaction of modified reagent with copper compounds - malachite, azurite and chrysocolla have been studied:

$$
\begin{gathered}
\mathrm{Cu}_{2} \mathrm{CO}_{3}(\mathrm{OH})_{2 \text { (solid) }}+2\left(\mathrm{NH}_{4}\right)_{2} \mathrm{~S}_{5 \text { (solid) }}=2 \mathrm{CuS}_{\text {(solid) }}+8 \mathrm{~S}_{\text {(solid) }}+\left(\mathrm{NH}_{4}\right)_{2} \mathrm{CO}_{3 \text { (solid) }}+2 \mathrm{NH}_{4} \mathrm{OH}_{\text {(liquid) }} ; \\
\mathrm{Cu}_{3}\left(\mathrm{CO}_{3}\right)_{2}(\mathrm{OH})_{2 \text { (solid) }}+3\left(\mathrm{NH}_{4}\right)_{2} \mathrm{~S}_{5 \text { (solid) }}=3 \mathrm{CuS}_{\text {(solid) }}+12 \mathrm{~S}_{\text {(solid) }}+2\left(\mathrm{NH}_{4}\right)_{2} \mathrm{CO}_{3 \text { (solid) }}+2 \mathrm{NH}_{4} \mathrm{OH}_{\text {(liquid) }} ; \\
\mathrm{CuSiO}_{3}\left(\mathrm{H}_{2} \mathrm{O}\right)_{2 \text { (solid) }}+\left(\mathrm{NH}_{4}\right)_{2} \mathrm{~S}_{5 \text { (solid) }}=\mathrm{CuS}_{\text {(solid) }}+4 \mathrm{~S}_{\text {(solid) }}+\left(\mathrm{NH}_{4}\right)_{2} \mathrm{SiO}_{3 \text { (solid) }}+2 \mathrm{H}_{2} \mathrm{O}_{\text {(liquid) }} .
\end{gathered}
$$

Probability of reactions was estimated by alteration of standard Gibbs energy.

Calculation of temperature dependence of standard Gibbs energy is based on Hess law [10] and was made using the formula

$$
\Delta_{\mathrm{r}} \mathrm{G}_{\mathrm{T}}^{0}=\sum \Delta_{\mathrm{f}} \mathrm{G}_{\mathrm{T}}^{0} \text { (products) }+\sum \Delta_{\mathrm{f}} \mathrm{G}_{\mathrm{T}}^{0} \text { (init.), }
$$

where $\Delta_{\mathrm{r}} \mathrm{G}^{0}{ }_{\mathrm{T}}$ - standard Gibbs energy at temperature (T), $\sum \Delta_{\mathrm{r}} \mathrm{G}^{0} \mathrm{~T}$ (products) — sum of standard Gibbs energy of reactions products formation at temperature (T), $\sum \Delta_{\mathrm{f}} \mathrm{G}^{0}{ }_{\mathrm{T}}$ (init.) - sum of standard Gibbs energy of initial components formation at temperature $(\mathrm{T})$. 
Temperature dependence of reaction velocity constant was calculated using the next formula:

$$
\lg K_{\mathrm{P}(\mathrm{T})}=-\Delta_{\mathrm{f}} \mathrm{G}_{\mathrm{T}}^{0} /(2.303 \mathrm{RT}),
$$

where $\mathrm{R}$ is the universal gas constant.

Temperature dependence of standard Gibbs energy of reactions products formation was determined by approximated method of Temkin-Schwartzman [11, 12], taking $\Delta \mathrm{Cp} 0=$ const, by the formula

$$
\Delta_{\mathrm{f}} \mathrm{G}_{\mathrm{T}}^{0}=\Delta_{\mathrm{f}} \mathrm{H}^{0}{ }_{298.15}-\mathrm{T} \Delta_{\mathrm{f}} \mathrm{S}^{0}{ }_{298.15}-\mathrm{TM} \Delta_{\mathrm{f}} \mathrm{C}_{\mathrm{p}}{ }_{298.15},
$$

where $\Delta_{\mathrm{f}} \mathrm{H}^{0}{ }_{298.15}$ is the standard enthalpy of compound formation at $298.15 \mathrm{~K} ; \Delta_{\mathrm{f}} \mathrm{S}^{0}{ }_{298.15}$ is the standard entropy of compound formation at $298.15 \mathrm{~K} ; \Delta_{\mathrm{f}} \mathrm{C}_{\mathrm{p}}{ }^{0} 298.15$ is the standard heat of compound formation at $298.15 \mathrm{~K}$; $\mathrm{M}$ is the coefficient, equal to

$$
\mathrm{M}=298,15 / \mathrm{T}-1+\ln (\mathrm{T} / 298.15) .
$$

Standard entropy was determined by formula

$$
\Delta_{\mathrm{f}} \mathrm{S}_{298,15}^{0}=\mathrm{S}_{298,15}^{0}-\sum \mathrm{S}^{0}{ }_{298,15} \text { (elementary substance), }
$$

where $\mathrm{S}_{298,15}^{0}$ is the standard entropy of compound at $298.15 \mathrm{~K} ; \sum \mathrm{S}_{298,15}^{0}$ (elementary substance) is the sum of standard entropies of substances forming the compound at $298,15 \mathrm{~K}$.

Standard heat capacity of compound formation was determined by the formula:

$$
\Delta_{\mathrm{f}} \mathrm{C}_{\mathrm{p}}{ }^{0} 298,15=\mathrm{C}_{\mathrm{p}}{ }^{0} 298,15-\sum \mathrm{C}_{\mathrm{p}}{ }_{298,15} \text { (elementary substance), }
$$

where $\mathrm{C}_{\mathrm{p}}{ }_{298,15}$ is the standard heat capacity of compound at $298,15 \mathrm{~K} ; \sum \mathrm{C}_{\mathrm{p}}{ }_{298,15}$ (elementary substance) is the sum of standard heat capacities of substances forming the compound, at $298.15 \mathrm{~K}$.

In case of insufficiency of the available literature data, the necessary values have been determined by proximate methods. tem:

Standard heat capacity was estimated by the Kumok method [13] based on heat capacity increments sys-

$$
\mathrm{C}_{\mathrm{p}}^{0}{ }_{298,15}\left(\mathrm{~A}_{\mathrm{m}} \mathrm{B}_{\mathrm{n}}\right)=\mathrm{mC}_{\mathrm{p}}{ }^{\mathrm{i}} 298,15\left(\mathrm{~A}^{\mathrm{n}+}\right)+\mathrm{nC}_{\mathrm{p}}{ }^{\mathrm{i}} 298,15\left(\mathrm{~B}^{\mathrm{m}-}\right)
$$

where $\mathrm{C}_{\mathrm{p}}{ }_{298,15}\left(\mathrm{~A}^{\mathrm{n}+}\right)$ is the increment of cation heat capacity; $\mathrm{C}_{\mathrm{p}}{ }^{\mathrm{i}} 298,15\left(\mathrm{~B}^{\mathrm{m}-}\right)$ is the increment of anion heat capacity.

\section{Results and Discussion}

The standard enthalpy values of compound formation at $298.15 \mathrm{~K}$ are given in the Table 1 . Values for $\mathrm{H}_{2} \mathrm{O}_{\text {(liquid) }}, \mathrm{NH}_{4} \mathrm{OH}_{\text {(liquid), }},\left(\mathrm{NH}_{4}\right)_{2} \mathrm{CO}_{3 \text { (solid), }} \mathrm{CuS}_{\text {(solid) }}, \mathrm{Cu}_{2} \mathrm{CO}_{3}[\mathrm{OH}]_{2 \text { (solid) }}, \mathrm{Cu}_{3}\left[\mathrm{CO}_{3}\right]_{2}[\mathrm{OH}]_{2 \text { (solid) }}$ compounds are from [6-8]. $\mathrm{S}_{\text {(solid) }}$ equals to $\mathrm{S}_{8(\mathrm{~g})}$ with enthalpy data by [14]. Standard enthalpy for $\left(\mathrm{NH}_{4}\right)_{2} \mathrm{~S}_{5 \text { (solid) }},\left(\mathrm{NH}_{4}\right)_{2} \mathrm{CO}_{3 \text { (solid) }}$ and $\left(\mathrm{NH}_{4}\right)_{2} \mathrm{SiO}_{3 \text { (solid) }}$ was calculated by Kassenov method from ionic increments [12]. Lacking data on increment of $\mathrm{S}_{5}{ }^{2-} \mathrm{SiO}_{3}{ }^{2-}$ ions were found by evaluation method using standard enthalpy of sodium pentasulfide and silicates of First Group metals [16]. Data for $\mathrm{CuSiO}_{3} \cdot \mathrm{nH}_{2} \mathrm{O}_{\text {(solid) }}$ are from [18].

Table 1

\section{Standard enthalpy of compound formation}

\begin{tabular}{|l|c|}
\hline \multicolumn{1}{|c|}{ Substance } & $-\Delta_{\mathrm{f}} \mathrm{H}^{0}{ }_{298,15}, \mathrm{~kJ} \cdot \mathrm{mol}^{-1}$ \\
\hline $\mathrm{H}_{2} \mathrm{O}_{\text {(liquid) }}$ & 285.829 \\
\hline $\mathrm{NH}_{4} \mathrm{OH}_{\text {(liquid) }}$ & 361.271 \\
\hline $\mathrm{S}_{\text {(solid) }}$ & 12.735 \\
\hline$\left(\mathrm{NH}_{4}\right)_{2} \mathrm{~S}_{5 \text { (solid) }}$ & 94.9 \\
\hline$\left(\mathrm{NH}_{4}\right)_{2} \mathrm{CO}_{3 \text { (solid) }}$ & 821.1 \\
\hline $\mathrm{CuS}_{\text {(solid) }}$ & 53.136 \\
\hline$\left(\mathrm{NH}_{4}\right)_{2} \mathrm{SiO}_{3 \text { (solid) }}$ & 1251.6 \\
\hline $\mathrm{Cu}_{2} \mathrm{CO}_{3}[\mathrm{OH}]_{2 \text { (solid) }}$ & 1051.020 \\
\hline $\mathrm{Cu}_{3}\left[\mathrm{CO}_{3}\right]_{2}[\mathrm{OH}]_{2 \text { (solid) }}$ & 1631.341 \\
\hline $\mathrm{CuSiO}_{3} \cdot \mathrm{nH}_{2} \mathrm{O}_{\text {(solid) }}$ & 1747.3 \\
\hline \multicolumn{2}{|l}{} \\
\hline
\end{tabular}

There are standard entropy values of compound formation at $298.15 \mathrm{~K}$ in the Table 2.

Data for $\mathrm{H}_{2} \mathrm{O}_{\text {(liquid) }}, \mathrm{NH}_{4} \mathrm{OH}_{\text {(liquid) }}, \mathrm{S}_{\text {(solid) }}, \mathrm{CuS}_{\text {(solid) }}, \mathrm{Cu}_{2} \mathrm{CO}_{3}[\mathrm{OH}]_{2 \text { (solid) }}, \mathrm{Cu}_{3}\left[\mathrm{CO}_{3}\right]_{2}[\mathrm{OH}]_{2 \text { (solid), }}, \mathrm{Cu}$ $\mathrm{SiO}_{3} \cdot \mathrm{nH}_{2} \mathrm{O}_{\text {(solid) }}$ were calculated by formula (8) based on [14-17]. For chrysocolla accepted $\mathrm{n}=2$. Standard 
entropy at $298.15 \mathrm{~K}$ for $\left(\mathrm{NH}_{4}\right)_{2} \mathrm{~S}_{5 \text { (solid) }},\left(\mathrm{NH}_{4}\right)_{2} \mathrm{CO}_{3 \text { (solid) }},\left(\mathrm{NH}_{4}\right)_{2} \mathrm{SiO}_{3 \text { (solid) }}$ was determined using entropy data found by Kumok method [13].

Table 2

Standard entropy of compound formation

\begin{tabular}{|l|c|}
\hline \multicolumn{1}{|c|}{ Substance } & $\Delta_{\mathrm{f}} \mathrm{S}^{0}{ }_{298,15}, \mathrm{~J} \cdot \mathrm{mol}^{-1} \cdot \mathrm{K}^{-1}$ \\
\hline $\mathrm{H}_{2} \mathrm{O}_{\text {(liquid) }}$ & -162.955 \\
\hline $\mathrm{NH}_{4} \mathrm{OH}_{\text {(liquid) }}$ & -359.004 \\
\hline $\mathrm{S}_{\text {(solid) }}$ & 23.567 \\
\hline$\left(\mathrm{NH}_{4}\right)_{2} \mathrm{~S}_{5 \text { (solid) }}$ & -579.1 \\
\hline$\left(\mathrm{NH}_{4}\right)_{2} \mathrm{CO}_{3 \text { (solid) }}$ & -841.3 \\
\hline $\mathrm{CuS}_{\text {(solid) }}$ & 1.453 \\
\hline$\left(\mathrm{NH}_{4}\right)_{2} \mathrm{SiO}_{3 \text { (solid) }}$ & -857.5 \\
\hline $\mathrm{Cu}_{2} \mathrm{CO}_{3}\left[\mathrm{OH}_{2(\text { solid) }}\right.$ & -503.504 \\
\hline $\mathrm{Cu}_{3}\left[\mathrm{CO}_{3}\right]_{2}[\mathrm{OH}]_{2 \text { (solid) }}$ & -671.479 \\
\hline $\mathrm{CuSiO}_{3} \cdot \mathrm{nH}_{2} \mathrm{O}_{\text {(solid) }}$ & -629.305 \\
\hline
\end{tabular}

Standard heat capacity of compounds formation at $298,15 \mathrm{~K}$ is shown in the Table 3.

For $\mathrm{H}_{2} \mathrm{O}_{\text {(liquid) }}, \mathrm{NH}_{4} \mathrm{OH}_{\text {(liquid) }}, \mathrm{S}_{\text {(solid) }}, \mathrm{CuS}_{\text {(solid) }}, \mathrm{Cu}_{2} \mathrm{CO}_{3}[\mathrm{OH}]_{2 \text { (solid) }}$ compounds the data were taken from [1417]. Values for $\left(\mathrm{NH}_{4}\right)_{2} \mathrm{~S}_{5 \text { (solid), }},\left(\mathrm{NH}_{4}\right)_{2} \mathrm{CO}_{3 \text { (solid) }},\left(\mathrm{NH}_{4}\right)_{2} \mathrm{SiO}_{3 \text { (solid) }}$ compounds were found using Kumok method [13], those for $\mathrm{CuSiO}_{3} \cdot \mathrm{nH}_{2} \mathrm{O}_{\text {(solid) }}$ are from [19].

Table 3

Standard heat capacity of compounds formation

\begin{tabular}{|l|c|}
\hline \multicolumn{1}{|c|}{ Substance } & $\Delta_{\mathrm{f}} \mathrm{C}_{\mathrm{p}}{ }^{0} 298.15 \cdot \mathrm{J} \cdot \mathrm{mol}^{-1} \cdot \mathrm{K}^{-1}$ \\
\hline $\mathrm{H}_{2} \mathrm{O}_{\text {(liquid) }}$ & 31.793 \\
\hline $\mathrm{NH}_{4} \mathrm{OH}_{\text {(liquid) }}$ & 53.576 \\
\hline $\mathrm{S}_{\text {(solid) }}$ & -2.594 \\
\hline$\left(\mathrm{NH}_{4}\right)_{2} \mathrm{~S}_{5 \text { (solid) }}$ & -17.633 \\
\hline$\left(\mathrm{NH}_{4}\right)_{2} \mathrm{CO}_{3 \text { (solid) }}$ & -14.3 \\
\hline $\mathrm{CuS}_{(\text {solid) }}$ & 0.712 \\
\hline$\left(\mathrm{NH}_{4}\right)_{2} \mathrm{SiO}_{3 \text { (solid) }}$ & -18.8 \\
\hline $\mathrm{Cu}_{2} \mathrm{CO}_{3}[\mathrm{OH}]_{2 \text { (solid) }}$ & -5.309 \\
\hline $\mathrm{Cu}_{3}\left[\mathrm{CO}_{3}\right]_{2}[\mathrm{OH}]_{2 \text { (solid) }}$ & 4.203 \\
\hline $\mathrm{CuSiO}_{3} \cdot \mathrm{nH}_{2} \mathrm{O}_{\text {(solid) }}$ & 45.288 \\
\hline
\end{tabular}

By means of the equation 6 the temperature dependence of standard Gibbs energy for every interacting compound have been calculated. The results are given in the Table 4.

Table 4

Temperature dependence of standard Gibbs energy of compounds formation

\begin{tabular}{|c|c|c|c|c|c|c|}
\hline \multirow{3}{*}{ Substance } & \multicolumn{6}{|c|}{$\mathrm{T}, \mathrm{K}$} \\
\hline & 298.15 & 313 & 328 & 343 & 358 & 373 \\
\hline & \multicolumn{6}{|c|}{$\Delta_{\mathrm{f}} \mathrm{G}_{\mathrm{T}}^{0}, \mathrm{~kJ} \cdot \mathrm{mol}^{-1}$} \\
\hline $\mathrm{H}_{2} \mathrm{O}_{\text {(liquid) }}$ & -237.244 & -234.836 & -232.426 & -230.038 & -227.67 & -225.323 \\
\hline $\mathrm{NH}_{4} \mathrm{OH}_{\text {(liquid) }}$ & -254.234 & -248.922 & -243.595 & -238.305 & -233.05 & -227.828 \\
\hline$S_{\text {(solid) }}$ & 5.708 & 5.359 & 5.009 & 4.660 & 4.313 & 3.967 \\
\hline$\left(\mathrm{NH}_{4}\right)_{2} \mathrm{~S}_{5 \text { (solid) }}$ & 77.759 & 86.365 & 95.070 & 103.788 & 112.517 & 121.258 \\
\hline$\left(\mathrm{NH}_{4}\right)_{2} \mathrm{CO}_{3 \text { (solid) }}$ & -570.266 & -557.768 & -545.133 & -532.488 & -519.834 & -507.171 \\
\hline $\mathrm{CuS}_{\text {(solid) }}$ & -53.569 & -53.591 & -53.614 & -53.637 & -53.66 & -53.684 \\
\hline$\left(\mathrm{NH}_{4}\right)_{2} \mathrm{SiO}_{3 \text { (solid) }}$ & -995.936 & -983.196 & -970.313 & -957.417 & -944.509 & -931.589 \\
\hline $\mathrm{Cu}_{2} \mathrm{CO}_{3}[\mathrm{OH}]_{2 \text { (solid) }}$ & -900.9 & -893.423 & -885.869 & -878.301 & -870.736 & -863.167 \\
\hline $\mathrm{Cu}_{3}\left[\mathrm{CO}_{3}\right]_{2}[\mathrm{OH}]_{2 \text { (solid) }}$ & -1431.139 & -1421.166 & -1411.09 & -1401.01 & -1390.928 & -1380.843 \\
\hline $\mathrm{CuSiO}_{3} \cdot 2 \mathrm{H}_{2} \mathrm{O}_{\text {(solid) }}$ & -1559.673 & -1550.344 & -1540.953 & -1531.594 & -1522.264 & -1512.963 \\
\hline
\end{tabular}


Using respective data from Table 4 in the equation (4) the temperature dependence of standard Gibbs energy for the reactions (1-3) was determined. Formula (5) was used to calculate temperature dependence of equilibrium constant for the reaction (Table 5).

Table 5

Temperature dependence of standard Gibbs energy and equilibrium constant

\begin{tabular}{|c|c|c|c|c|c|c|c|}
\hline Reaction & $\mathrm{T}, \mathrm{K}$ & 298.15 & 313 & 328 & 343 & 358 & 373 \\
\hline \multirow{3}{*}{10} & $-\Delta_{\mathrm{r}} \mathrm{G}^{0} \mathrm{~T} \cdot \mathrm{kJ} \cdot \mathrm{mol}^{-1}$ & 394.826 & 399.229 & 403.75 & 408.367 & 413.048 & 417.808 \\
\hline & Equation & \multicolumn{6}{|c|}{$\Delta_{\mathrm{r}} \mathrm{G}_{\mathrm{T}}^{0}=-0.307 \mathrm{~T}-303.1$} \\
\hline & $\lg K_{P}$ & 69.25 & 66.70 & 64.37 & 62.26 & 60.34 & 58.58 \\
\hline \multirow{3}{*}{11} & $-\Delta_{\mathrm{r}} \mathrm{G}^{0} \mathrm{~T} \cdot \mathrm{kJ} \cdot \mathrm{mol}^{-1}$ & 543.349 & 547.774 & 552.31 & 556.931 & 561.615 & 566.377 \\
\hline & Equation & \multicolumn{6}{|c|}{$\Delta_{\mathrm{r}} \mathrm{G}_{\mathrm{T}}^{0}=-0.307 \mathrm{~T}-451.5$} \\
\hline & $\lg K_{P}$ & 95.30 & 91.52 & 88.06 & 84.91 & 82.04 & 79.41 \\
\hline \multirow{3}{*}{12} & $-\Delta_{\mathrm{r}} \mathrm{G}^{0} \mathrm{~T} \cdot \mathrm{kJ} \cdot \mathrm{mol}^{-1}$ & 19.247 & 21.044 & 22.86 & 24.684 & 26.51 & 28.346 \\
\hline & Equation & \multicolumn{6}{|c|}{$\Delta_{\mathrm{r}} \mathrm{G}_{\mathrm{T}}^{0}=-0.121 \mathrm{~T}+16.99$} \\
\hline & $\lg K_{P}$ & 3.38 & 3.52 & 3.64 & 3.76 & 3.87 & 3.97 \\
\hline
\end{tabular}

Based on Table 5 data, the temperature dependence diagrams were built for interaction of malachite, azurite and chrysocolla with modified reagent (Fig. 1).


Figure 1. Temperature dependence of standard Gibbs energy for interaction of copper minerals with modified reagent

As follows from Figure 1, standard Gibbs energy for malachite and azurite is negative in the whole temperature range, indicating high probability of interaction, growing with temperature. Interaction with chrysocolla is less active than with malachite and azurite but is still possible at mentioned temperature, with interaction probability again proportional to temperature growth.

Interaction of a modified reagent with chrysocolla in interval of $298.15-373 \mathrm{~K}$ is lower than for malachite and azurite. But also it can proceed in this temperature interval. Raise of temperature also is able to increase the probability of interaction. 


\title{
Conclusions
}

Based on the results of thermodynamic analysis the modified reagent is recommended as oxidized copper minerals sulphidizer for flotation enrichment of oxidized copper ore. In particular, the possibility of sulphidizing the ore containing malachite, azurite and chrysocolla as major components, is demonstrated in the study.

Financial support of scientific research was carried out under the project AP 05130454 «Development of an effective technology for the processing of oxidized copper ores using a modified reagent» of the Ministry of education and sciences of the Republic of Kazakhstan.

\section{References}

1 Митрофанов С.И. Исследование руд на обогатимость / С.И. Митрофанов. - М.: Металлургиздат, 1954. - 23 с.

2 Feng Q.C. Effect of reagent Regime on Flotation Performance of Refractory Oxidized Copper Ores Containing a High Content of Slime / Q.C. Feng, S.M. Wen, W.J. Zhao, C.Y. Chen, H.F. Zhao, Y.J. Wang // Advanced Materials Research. - 2013. - P. 23512355 .

3 Elgillani D.A. Classroom notes on Surface Chemistry and Flotation / D.A. Elgillani. — Dept. of Mining Engineering, Faculty of Engineering, Cairo University, 2008.

4 Corina K.C. The recovery of oxide copper minerals from a complex copper ore by sulfidization / K.C. Corina, M.O. Kalichinia, C.T. Connora, S. Simukanga // Minerals Engineering. - 2017. — P. 15-17.

5 Бектурганов Н.С. Применение сульфидизации при переработке окисленных медных руд Удоканского месторождения / Н.С. Бектурганов, Г.Л. Каткеева, И.М. Оскембеков, М.А. Акубаева // Цветные металлы. — 2016. — № 9. — С. $22-27$.

6 Каткеева Г.Л. Изучение влияния электрохимической обработки на сульфидизацию и флотацию окисленных медных минералов и руд / Г.Л. Каткеева, Н.С. Бектурганов, З.Б. Сагиндыкова и др. // Обогащение руд. - 2004. - № 6- С. $20-22$.

7 Бектурганов Н.С. Физико-химические особенности процесса гидротермального сульфидирования окисленных и смешанных видов сырья цветной металлургии / Н.С. Бектурганов, А.К. Базаев, С.П. Сим и др. // Сб. науч. тр. АН СССР. - 1989. - C. 51-59.

8 Патент № 30898 РК, МПК С22В 15/00. Способ обогащения окисленных медных руд / И.М. Оскембеков, Н.С. Бектурганов, Г.Л. Каткеева, Ж.С. Оскембекова, Ж.А. Шайке. — Опубл. 15.02.2016. — Бюл. — № 2.

9 Патент № 32695 РК, МПК В03В 7/00. Способ обогащения окисленной медной руды / И.М. Оскембеков, Н.С. Бектурганов, Г.Л. Каткеева, Ж.С. Оскембекова, М.А. Акубаева, Д.Р. Гизатуллина, Ж.А. Шайке, Е.М. Жунусов, А.М. Жунусов. Опубл. 05.03. 2018. - Бюл. — № 9.

10 Герасимов Я.И. Курс физической химии. - Т. І. / Я.И. Герасимов. — М.: Госхимиздат, 1963. - 624 с. $392 \mathrm{c}$.

11 Рябин В.А. Термодинамические свойства веществ / В.А. Рябин, М.А. Остроумов, Т.Ф. Свит. - Л.: Химия, 1977. -

12 Касенов Б.К. Термодинамические методы в химии и металлургии / Б.К. Касенов, А.С. Пашинкин, М.К. Алдабергенов. - Алматы: Рауан, 1994. — 256 с.

13 Кумок В.Н. Проблема согласования методов оценки термодинамических характеристик. Прямые и обратные задачи химической термодинамики / В.Н. Кумок. - Новосибирск: Наука, 1987. - С. 108-123.

14 Термические константы веществ: справоч. - Вып. І / под ред. В.П. Глушко. — М.: Наука, 1965.— 146 с.

15 Термические константы веществ: справоч. - Вып. VI / под ред. В.П. Глушко. — М.: Наука, 1972.— 370 с.

16 Термические константы веществ: справоч. — Вып. ІІІ / под ред. В.П. Глушко. — М.: Наука, 1968.-222 с.

17 Термические константы веществ: справоч. - Вып. II / под ред. В.П. Глушко. — М.: Наука, 1966. — 96 с.

18 Термические константы веществ: справоч. - Вып. IV / под ред. В.П. Глушко. — М.: Наука, 1970. — 510 с.

19 Сайт журнала «Свойства минералов». [Электронный ресурс]. — Режим доступа: http://www.74rif.ru/mineral-gl.html.

\section{Г.Л. Каткеева, Г. Бүркітсетерқызы, Ю.П. Морозов, Е.М. Жунусов}

\section{Тотыққан мыс минералдарының модификацияланған реагентпен әрекеттесуінің термодинамикалық талдауы}

\begin{abstract}
Тотыққан мыс кендерін байыту кезінде тотыққан мыс минералдарын алдын ала сульфидтеу керектігі көрсетілген. Алғаш рет тотыққан мыс кендерінің негізін құраушы - малахит, азурит және хризоколланың модификацияланған сульфидтеуші реагентпен әрекеттесуінің термодинамикалық талдауы жүргізілді. Модификацияланған сульфидтеуші реагент ретінде натрий полисульфиді мен аммоний сульфатының ерітіндісінде пайда болған аммоний полисульфиді қолданылды. Аммоний полисульфидінің тотыққан мыс минералдарымен реакциясының стандартты Гиббс энергиясының температуралық тәуелділігі айқындалды. Малахит пен азурит үшін 298,15-373 К аралығында модификацияланған реагентпен реакциясы кезінде стандартты Гиббс энергиясы теріс әсер көрсететіндігі анықталды. Сондықтан модификацияланған реагенттің малахит және азуритпен әрекеттесу ықтималдығы жоғары. Температураның
\end{abstract}


жоғарылауы өзара әсер ету ықтималдығын арттырады. Модификацияланған реагенттің хризоколламен әрекеттесуі малахит пен азуритке қарағанда әлсіз көрсетілген. Алайда осы температура интервалында ағып кетуі мүмкін. Температураның жоғарылауы сонымен қатар өзара әсер ету ықтималдылығын арттырады. Осыған байланысты, тотыққан мыс минералдарының модификацияланған сульфидтеуші реагенті - аммоний полисульфидімен сульфидтенуінің мүмкіндігі көрсетілген.

Кілт сөздері: тотыққан минералдар, хризокола, малахит, азурит, сульфидтеуші модификацияланған реагент, сульфидтеу, термодинамикалық талдау, Гиббс энергиясы.

\title{
Г.Л. Каткеева, Г. Буркитсетеркызы, Ю.П. Морозов, Е.М. Жунусов
}

\section{Термодинамический анализ взаимодействия окисленных минералов меди с модифицированным реагентом}

\begin{abstract}
Показана необходимость предварительной сульфидизации окисленных минералов меди при обогащении окисленной медной руды. Впервые проведен термодинамический анализ взаимодействия модифицированного сульфидирующего реагента с основными составляющими окисленной медной руды - малахитом, азуритом и хризоколлой. В качестве модифицированного сульфидирующего реагента применен полисульфид аммония, образованный в растворе полисульфида натрия и сульфата аммония. Определена температурная зависимость стандартной энергии Гиббса реакции полисульфида аммония с окисленными медными минералами. Показано, что изменение стандартной энергии Гиббса реакции для малахита и азурита с модифицированным реагентом в интервале 298,15-373 К принимает отрицательные значения. Следовательно, высока вероятность взаимодействия модифицированного реагента с малахитом и азуритом. Повышение температуры способствует увеличению вероятности взаимодействия. Связь модифицированного реагента с хризоколлой выражена слабее, чем малахита и азурита. Однако она может протекать в данном температурном интервале. Повышение температуры также способствует увеличению вероятности взаимодействия. Таким образом, установлена принципиальная возможность сульфидизации окисленных медных минералов модифицированным сульфидирующим реагентом - полисульфидом аммония.
\end{abstract}

Ключевые слова: окисленные минералы, хризоколла, малахит, азурит, модифицированный сульфидирующий реагент, сульфидизация, термодинамический анализ, энергия Гиббса.

\section{References}

1 Mitrofanov, S.I. (1954). Issledovanie rud na obohatimost [Ore research on enrichment]. Moscow: Metallurhizdat [in Russian].

2 Feng, Q.C., Wen, S.M., Zhao, W.J., Chen, C.Y., Zhao, H.F. \& Wang, Y.J. (2013). Effect of reagent Regime on Flotation Performance of Refractory Oxidized Copper Ores Containing a High Content of Slime. Advanced Materials Research, 2351-2355.

3 Elgillani, D.A. (2008). Classroom notes on Surface Chemistry and Flotation. Department of Mining Engineering, Faculty of Engineering, Cairo University.

4 Corina, K.C., Kalichinia, M.O., Connora, C.T. \& Simukanga, S. (2017). The recovery of oxide copper minerals from a complex copper ore by sulfidization. Minerals Engineering, 15-17.

5 Bekturganov, N.S., Katkeeva, G.L., Oskembekov, I.M. \& Akubaeva, M.A. (2016). Primenenie sulfidizatsii pri pererabotke okislennykh mednykh rud Udokanskoho mestorozhdeniia [The use of sulfidization in the processing of oxidized copper ores of the Udokan deposit]. Tsvetnye metally - Non-ferrous metals, 9, 22-27 [in Russian].

6 Katkeeva, G.L., Bekturganov, N.S. \& Sagindyikova, Z.B. et al. (2004). Izuchenie vliianiia elektrokhimicheskoi obrabotki na sulfidizatsiiu i flotatsiiu okislennykh mednykh mineralov i rud [Study of the effect of electrochemical treatment on the sulfidization and flotation of oxidized copper minerals and ores]. Obohashchenie rud-Ores enrichment, 6, 20-22 [in Russian].

7 Bekturganov, N.S., Bazaev, A.K. \& Sim, S.P. et al. (1989). Fiziko-khimicheskie osobennosti protsessa hidrotermalnoho sulfidirovaniia okislennykh i smeshannykh vidov syria tsvetnoi metallurhii [Physico-chemical characteristics of the process of hydrothermal sulfidation of oxidized and mixed raw materials of non-ferrous metallurgy]. Sbornik nauchnykh trudov AN SSSR - Collection of scientific papers of the USSR Academy of Sciences, 51-59 [in Russian].

8 Oskembekov, I.M., Bekturganov, N.S., Katkeeva, G.L., Oskembekova, Zh.S. \& Shayke, Zh.A. (2016). Sposob obohashcheniia okislennykh mednykh rud [A method of enrichment of oxidized copper ores]. Patent No. 30898 RK, MPK S22 V 15/00. Publ. BI, 2 [in Russian].

9 Oskembekov, I.M, Bekturganov, N.S, Katkeeva, G.L, Oskembekova, Zh.S., Akubaeva, M.A., \& Gizatullina, D.R., et al. (2018). Sposob obohashcheniia okislennoi mednoi rudy [A method of enrichment of oxidized copper ore]. Patent No. 32695 RK, MPK V03V 7/00. Publ. BI, 9 [in Russian].

10 Gerasimov, Ya.I. (1963). Kurs fizicheskoi khimii [Physical Chemistry Course]. (Vol. 1). Moscow: Hoskhimizdat [in Russian].

11 Ryabin, V.A., Ostroumov, M.A. \& Svit, T.F. (1977). Termodinamicheskie svoistva veshchestv [Thermodynamic properties of substances]. Leningrad: Khimiia [in Russian].

12 Kasenov, B.K., Pashinkin, A.S. \& Aldabergenov, M.K. (1994). Termodinamicheskie metody v khimii i metallurhii [Thermodynamic methods in chemistry and metallurgy]. Almaty: Rauan [in Russian]. 
13 Kumok, V.N. (1987). Problema sohlasovaniia metodov otsenki termodinamicheskikh kharakteristik. Priamye i obratnye zadachi khimicheskoi termodinamiki [The problem of harmonization of methods for assessing thermodynamic characteristics. Direct and inverse problems of chemical thermodynamics]. Novosibirsk: Nauka [in Russian].

14 Glushko, V.P. (Eds.) (1965). Termicheskie konstanty veshchestv: spravochnik [Thermal constants of substances. Directory]. (Iss. I). Moscow: Nauka, 146 [in Russian].

15 Glushko, V.P. (Eds.) (1972). Termicheskie konstanty veshchestv: spravochnik [Thermal constants of substances. Directory]. (Iss. VI). Moscow: Nauka, 370 [in Russian].

16 Glushko, V.P. (Eds.) (1968). Termicheskie konstanty veshchestv: spravochnik [Thermal constants of substances. Directory]. (Iss. III). Moscow: Nauka, 222 [in Russian].

17 Glushko, V.P. (Eds.) (1966). Termicheskie konstanty veshchestv: spravochnik [Thermal constants of substances. Directory]. (Iss. II). Moscow: Nauka, 96 [in Russian].

18 Glushko, V.P. (Eds.) (1970). Termicheskie konstanty veshchestv: spravochnik [Thermal constants of substances. Directory]. (Iss. IV). Moscow: Nauka, 510 [in Russian].

19 Sait zhurnalu «Svoistva mineralov» [Site of journal «Mineral Properties»]. www.74rif.ru. Retrieved from http://www.74rif.ru/mineral-gl.html [in Russian]. 